\title{
Predicting the Structural Performance of Heat-Treatable Al-Alloys
}

\author{
M.J. Starink, I. Sinclair, P.A.S Reed and P.J. Gregson \\ Materials Research Group, School of Engineering Sciences, \\ University of Southampton, Southampton S017 1BJ, UK
}

Keywords: $\quad$ aerospace alloys, physical modelling, adaptive numeric modelling, strength, toughness, conductivity.

\begin{abstract}
Advances in physically-based and adaptive numeric modelling (ANM) have lead to a significant elevation of the role of modelling in commercial alloy development and process optimisation. Within these two categories of modelling a wide variety of techniques exists, whilst hybrid approaches, taking advantage of the benefits of the both methods, are also being developed. In this paper various ANM techniques and physically-based models relevant for modelling and predicting the properties of heat treatable Al-based alloys are reviewed, and case studies involving the modelling of proof stress, toughness and conductivity of a range of Al-based alloys are presented. These case studies illustrate that successful modelling requires an in-depth understanding of the various modelling options. Selection of the optimum modelling approach is driven by a range of factors, such as size of database available (large databases are best analysed using adaptive numeric modelling approaches), and availability of sound physical understanding (the latter benefiting physically-based approaches).
\end{abstract}

\section{Introduction}

Throughout the history of alloy development, the modelling of properties and microstructure-property relationships has lagged behind the commercialisation of new products and processes. In terms of agehardened Al-alloys, commercial aerospace applications were established by 1919, however the first basic models of age hardening, based on dislocation movement and dislocation pinning, were not developed until the 1940's [1,2], whilst the first detailed models of strengthening in multi-component Al-based alloys were only published in the 1980/90s [3,4,5]. In recent years, this time lag has been reduced, with modelling preceding or directly leading product development in some cases. A specific example is the formulation of new compositions of Ni-based superalloys by Rolls-Royce Plc. which are based on thermodynamic modelling, and the recent development of 7449 and 7040 Al-alloy plate which has been influenced by microstructure-property modelling [6]. The use of well-founded modelling approaches has clear advantages in directing product development in a cost effective manner.

Whilst the above examples concern modelling through physical understanding of microstructures and microstructure development, modelling using adaptive numeric (AN) approaches such as artificial neural networks has recently made important advances. Current state-of-the-art adaptive numeric modelling combines flexible, adaptive algorithms for model construction with a rigorous mathematical/statistical basis $[7,8]$.

The purpose of the present paper is to highlight recent advances in physically-based and AN modelling, and to consider the relative benefits of the two approaches as well as hybrid approaches which use elements of both. In exploring this wide ranging and continuous field of modelling approaches we will start by introducing some of the better known techniques. On one side of the spectrum of modelling 
approaches, we find the purely physically-based approach in which models are derived using physical understanding of the processes, properties and/or microstructures without using any new data. This approach is only possible if we have a detailed understanding of the underlying mechanisms and if model parameters/physical constants are accurately known. Clearly, such idealised ab initio modelling will only be possible in a limited number of cases where prior research has clearly elucidated the underlying physics. If understanding is more limited and model parameters are not accurately known, we may use a semi-empirical approach where regression functions are estimated from basic physical understanding and experimental data is used to 'calibrate' the model (i.e. a regression is used to determine the unknown physical parameters). Such an approach may be expected to work well in cases where sound, but not complete/exact physical understanding of the underlying mechanisms can be obtained. Such semi-empirical and ab initio modelling may be termed "white box", in that the mathematical relationships between model inputs (materials parameters, test conditions etc.) and the output parameter (performance characteristic) are reasonably clear and may be linked to mechanistic understanding. For more complex situations, with many interrelated variables and where the system understanding is limited, unreliable or non-existent, adaptive numeric techniques may be used to examine data distributions carefully to extract a variety of mathematical relationships that may fit available data equally well, however significant problems may then arise in the identification of the "correct" mathematical relationship. A well-known example of adaptive numeric modelling is the use of artificial neural networks (ANNs) [9]. Here a flexible regression function is specified via a network architecture, with an appropriate learning algorithm then being used to set the weights of the network connections on the basis of available experimental data (a process known as 'training'). This is typically achieved by reducing the mean squared error (MSE) of the model, which is based on the error between model prediction values for a given set of input parameters and corresponding measured (or 'target') values. A certain proportion of the available data is generally used to train the network, whilst the remainder is reserved to test the model - thus assessing 'generalisation ability'. An overly complex model may fit the training data (which was used to construct the model) very well but will predict the test data very poorly as it has over-fitted the data (see Fig. 1c). Similarly an overly simple model will under-fit both the training and test data (see Fig. 1a). The optimum model will match the complexity of the underlying physical mechanism. ANNs may provide several sets of complex (usually nonlinear) mathematical relationships which equally well predict the observed data distributions. The underlying mechanisms are not easily visualised from these relationships (i.e. it is a "black box" method). Limited local transparency/trend information can be gleaned by systematically varying one input whilst maintaining all other inputs at a constant value and observing the effect on the predictions (i.e. producing a series of sections through the model space), although this process becomes more and more restricted as input dimensionality increases.

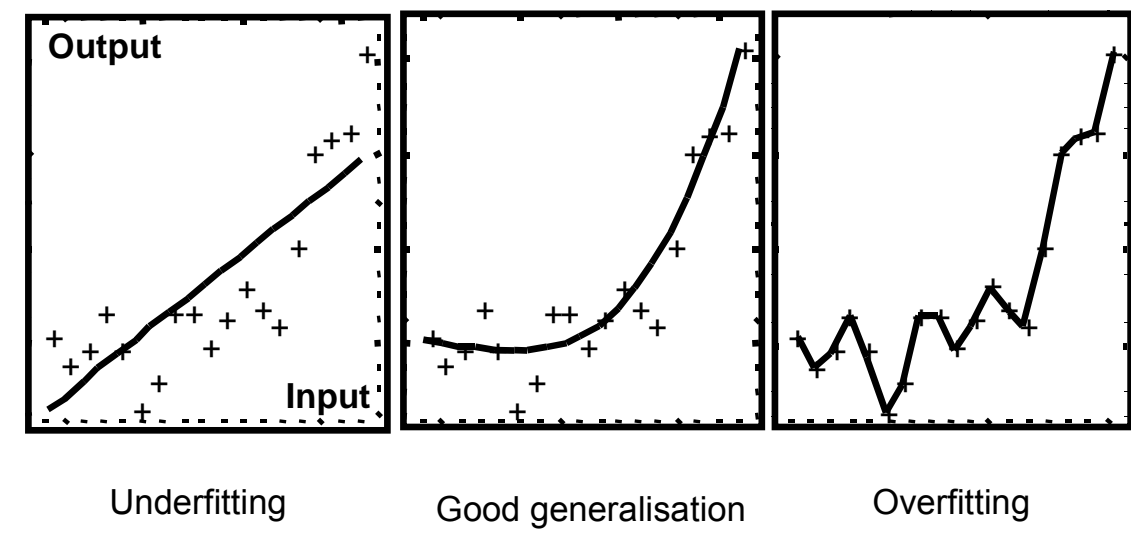

Fig. 1 (a) an overly simple linear fit to data (b) a good approximation to the underlying relationship (c) an overfitted model, where the training data has been precisely fitted, giving rise to an unrealistic relationship. 
A priori, an AN approach to modelling of data can be expected to provide a rational modelling strategy when i) the available data covers the relevant input parameters well, ii) a large amount of data is available, iii) underlying relationships are complex and iv) the data are noisy. As such, these methods may be well suited to modelling complex commercial processing operations where quantitative physical models may be poorly developed but extensive process-property databases are available. In terms of the amount of data necessary to establish a good model, guidelines are difficult. One should typically consider that for a complete assessment of all possible interrelated terms, the amount of data needed is an exponential function of the number of relevant input parameters. For instance in modelling $\mathrm{T} 7$ strengths of 7xxx alloys, considering only solution treatment and one-step ageing, at least 3 compositions ( $\mathrm{Zn}, \mathrm{Mg}, \mathrm{Cu}$ ), two temperatures (solution treatment, ageing), two isothermal ageing/solution treatment times and two heating/cooling rates should be contained in the data. Thus this example with 9 inputs would necessitate (as a very rough estimate) $2^{9}=512$ well distributed data points. Clearly, this may not be feasible. In general, AN modelling may have to be performed with data that is limited, or badly distributed, or both. This puts great demands on these AN modelling approaches, and may require additional strategies in our modelling e.g. the incorporation of some elements of physically-based knowledge (i.e. prior knowledge) into the system. If we can extract clear input-output trend information from AN models we can also use our metallurgical understanding to assess the validity of the models produced.

It is difficult to define the cases in which physically-based modelling can be expected to yield best results as deriving a physical model often depends on specific individual modelling skills and knowledge. Factors that indicate a physical modelling approach may be more appropriate than an AN approach are: i) availability/understanding of relevant microstructure-property mechanisms, ii) limited availability of data, iii) badly distributed data and iv) accurate (low noise) data.

\section{Modelling Approaches}

\section{$2.1 \quad$ Adaptive numeric modelling}

Adaptive numeric modelling can be defined as the extraction, by empirical mathematical means, of system relationships from observational data and thereby predicting the responses of that system. Ultimately the quantity and the quality of the observations govern the performance of the model constructed through this approach. In the context of industrial material science, often only partial knowledge is available about the physical processes involved, although significant amounts of 'raw' data may be available from production records and may be used to construct a data driven model. In modelling of properties and processing of Al-based alloys AN modelling has been applied to processing-property relations in $2 \mathrm{xxx}$ and $7 \mathrm{xxx}$ plate [7,8,10,11], fatigue life of 2xxx panels [12], air bending of a 5xxx alloy [13], damage evolution in MMCs [14], hot working [15] and welding [16].

In mathematical terms, AN modelling considers a dataset $D_{N}=\left\{x_{i}, y_{i}\right\}_{i=1}^{N}$, drawn from an unknown probability distribution, $F$, where $x_{i}$ represents a set of inputs (e.g. alloy composition and/or thermomechanical processing information), $y_{i}$ represents a set of outputs, (e.g. mechanical properties) and $N$ represents the number of data-points. The empirical modelling problem then is to find any underlying mapping $x \rightarrow y$ that is consistent with the dataset $D_{N}$. Typically, sampling of data is non-uniform and, due to the high dimensional nature of the problems of interest (i.e. large numbers of inputs), the data will only form a sparse distribution in the input space. To address the ill-posed [17] nature of the problem, it is necessary to convert the problem to one that is well posed. For the problem to be well posed a unique solution must exist that varies continuously with the data. In our group AN modelling is performed using a range of model construction strategies including Neurofuzzy approaches, Bayesian multi-layer perceptron (MLP) methods and Support Vector Machines (SVMs), whilst basic Multivariate Linear models are used as a benchmark. 


\section{The Multivariate Linear Model}

A multivariate linear model is given by:

$$
y=w_{0}+w_{1} x_{1}+w_{2} x_{2}+\ldots+w_{n} x_{n}
$$

where $x_{1}, \ldots, x_{n}$ is the models input vector, $w_{1}, \ldots, w_{n}$ are unknown weight parameters to be estimated, and $w_{0}$ is a bias term. The unknown vector of parameters, $w$, can be estimated together with the associated parametric uncertainty in the standard least squares sense [18].

\section{Neurofuzzy (NF) modelling}

NF networks [19] provide quantitative predictions in which dependencies on individual input parameters remain transparent, offering a balance of quantitative accuracy and qualitative insight into the behaviour of the system. In NF modelling a compromise between accuracy of the fit and complexity of the approximating functions must be sought. For this, the Structural Risk Minimisation [20] principle is adopted. The development of model structure is then based on the ANalysis Of VAriance (ANOVA) representation, i.e. the concept that any high dimensional function may be broken down into a subset of terms from the expansion:

$$
f(x)=f_{0}+\sum_{i=1}^{N} f_{i}\left(x_{i}\right)+\sum_{i=1}^{N} \sum_{j=1+1}^{N} f_{i, j}\left(x_{i}, x_{j}\right)+\ldots+f_{1,2, \ldots, N}(x)
$$

where $N$ represents the number of input parameters, $f_{0}$ is a constant (bias term) and the other terms on the right hand side represent all possible univariate, bivariate, trivariate, etc., functional combinations of the input parameters. Recent successful NF materials applications include prediction of Ni-based superalloy fatigue behaviour, with the superalloy fatigue work demonstrating that a combination of basic materials' properties and test conditions readily provided physically reasonable models of nearthreshold crack growth [21], whilst pure neural network models of equivalent data frequently exhibited unrealistic physical characteristics, engendering extensive model interrogation and verification processes [22].

\section{Bayesian Multi-layer Perceptron}

A Bayesian multi-layer perceptron (MLP) is a type of artificial neural network in which network training takes place using Bayesian learning [23]. An ANN is composed of many simple interconnected nodes (neurons) where the interconnections are "weighted" and these weights are adapted on the basis of example (training) data. ANNs employ techniques which have arisen in a number of different but related areas such as regression analysis, statistical inferencing, machine learning and computational learning theory. More details of ANNs and Bayesian MLPs are presented in Ref. [7]. Whilst ANNs in general can produce very accurate models, relations obtained are not easy to visualise, i.e. they are "black box" methods.

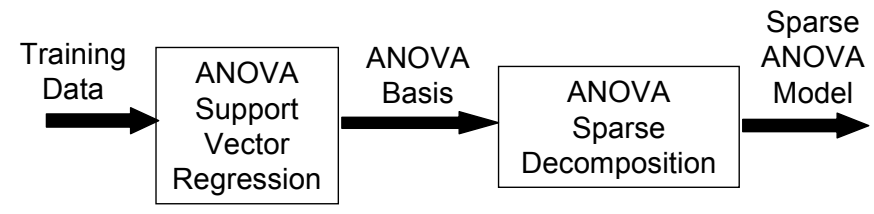

Data

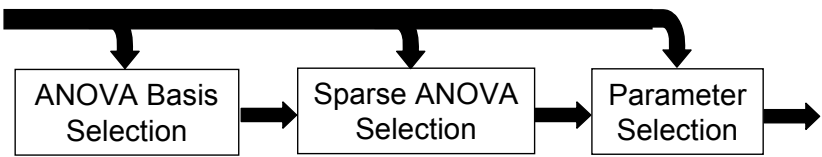

Fig. 2:

Schematic diagram illustrating the construction of a full ANOVA regression using the SVM approach and the subsequent sparse decomposition yielding the more transparent sparse ANOVA representation (SUPANOVA).

\section{Support Vector Machines (SVMs)}

SVMs have recently received an intensified research effort, due to many attractive features and promising empirical performance. The formulation embodies the principle of structural risk minimisation (SRM) developed by Vapnik [18]. SRM differs from the commonly used empirical risk 
minimisation (ERM) by trying to minimise an upper bound on the expected risk, rather than minimising the error on the training set. SVMs, like the Bayesian MLP, are essentially "black box" models, however, transparency can be introduced by use of the SUPANOVA framework [18]. The SUPANOVA promotes a sparse representation within an ANOVA (Analysis Of VAriance) representation (see Fig. 2). It describes the decomposition of the function into additive components, with the objective being to represent this function by a subset of terms from an expansion. For more details see Refs. [7,10].

\subsection{Physically-based approaches to modelling}

The main philosophy behind physical approaches to modelling is that the relationships between microstructure, physical processes and properties are captured in physically-based models which describe the underlying processes dominating the property under consideration. The physical processes and microstructural elements which determine properties of Al-based alloys are varied and often complex, and, hence, successful physical modelling in materials science generally requires a research effort in which specialist materials knowledge, materials data, mathematical/statistical analysis, computer/programming skills and creation of new models are integrated.

Well known examples of the physically-based approach relevant to aluminium alloy properties are thermodynamic modelling of equilibrium phases (see e.g. [24]), the modelling of strength of crystalline materials using theory of dislocation movement and dislocation pinning (by precipitates, grain boundaries) (e.g. [1,2,25]), as well as finite element (FE) modelling of mechanical properties (e.g. for composite material). In principle physically-based approaches can be adopted for any material property but ultimate success is often determined by current availability of reliable models and economics of model development. As an example of the latter it is noted that in materials development and materials processing industries, the application of thermodynamic modelling software has been limited mainly to larger companies (see e.g. [29]).

\subsection{Hybrid physical-AN approaches to modelling}

A hybrid approach can combine some benefits of the methods: faster assessment of large databases employing AN modelling and better understanding of the physics and the possibility of better extrapolation capability from the use of physical models. In exploring these hybrid approaches it is important to note that in many cases even approaches presented as being purely numeric will contain some elements of basic physical ideas/models through the selection and/or pre-processing of input variables and/or pre-processing of the target properties. This may be based on very simple physical understanding, e.g. precipitation is known to be thermally activated (basic physical understanding) and hence ageing temperature will be used as input (input selection) by measuring temperature on a calibrated temperature scale (basic physical understanding).

Hybrid approaches to modelling can take many forms, but in general they can be divided into three categories: i) data pre-processing using physically-based modelling approaches, ii) the use of functions derived in a physically-based model within the AN model optimisation algorithm and iii) AN modelling of residual errors from a physically-based modelling approach. In the present work we especially consider the pre-processing approach (i). This approach may be applied to just one input parameter; for example, a reaction which is considered to be thermally activated, generally an exponential dependence on temperature is expected (= physical understanding), thus measured temperatures can be transformed into $\log (\mathrm{T})$ to facilitate accurate model construction in subsequent AN modelling. In a more complex pre-processing approach $n_{\text {in }}$ measured input parameters can be combined to yield $\mathrm{n}_{\mathrm{pp}}$ new parameters which are considered to yield more physically valuable information. One example of this pre-processing is combining gross compositions and solution treatment temperature (original inputs) with phase diagram information (physical understanding) to 
calculate fractions of dissolved elements and amounts of undissolved intermetallic phases (preprocessed inputs) (see e.g. Ref. [8] and section 3.2).

\section{$3 \quad$ Modelling properties of aerospace alloys.}

\subsection{Case 1: Strength of 2xxx alloys}

In this case we consider an extensive commercial dataset for the Al-Cu-Mg-Mn aluminium alloy 2024 in a T351 temper, with the objective being to predict $0.2 \%$ proof stress under real commercial processing conditions. The pre-processed dataset of 290 data lines comprised ten characteristics; the final gauge (FG), $\mathrm{Cu}, \mathrm{Fe}, \mathrm{Mg}, \mathrm{Mn}, \mathrm{Si}$, cast slab length ( $\mathrm{SL})$, solution treatment time $\left(\mathrm{t}_{\text {sol }}\right)$, percentage stretch (\%st), and degree of cross-rolling (RR). Clearly, for this complex and highly-dimensional system no comprehensive physically-based model is available and AN modelling is the only viable route for extracting information from the data.

\section{AN modelling}

For this dataset Multivariate Linear models, Neurofuzzy approaches, Bayesian multi-layer perceptron (MLP) and Support Vector Machines (SVMs) were used, and results show that SVM modelling provides the best accuracy (i.e. the lowest test MSE), whilst representing reasonably well the strength variations across the available data range, see Fig. 3. In the full ANOVA representation for this dataset 1024 different functions/terms are possible, however through multiple data re-sampling (repartitioning) the SVM approach identified that only 19 terms were significant, with 13 terms appearing in virtually all the models - i.e. a high degree of mathematical consistency was achieved, independent of data partitioning. These include simple univariate functions of $\mathrm{Mg}, \mathrm{Si}$, $\mathrm{t}_{\text {sol }}$ and $\%$ st, as well as various bivariate and trivariate terms. The univariate terms reflect well-known (and some lesserknown) relations between composition and processing on the one hand and yield strength of $2 \mathrm{xxx}$ alloys on the other, e.g. percentage stretch and $\mathrm{Mg}$ content increasing strength through enhanced dislocation strengthening/work hardening and precipitation strengthening. As is further illustrated elsewhere [7,10], the combination of high modelling accuracy and transparency (the latter introduced by virtue of the SUPANOVA framework) makes the SVM modelling approach ideally suited for analysing the present type of highly dimensional, complex datasets, leading to process optimisation that can generally not be obtained by any other modelling technique, be it adaptive numeric or physically-based.

\subsection{Case 2: Conductivity of T7 7xxx alloys.}

In aerospace applications, high strength $7 \mathrm{xxx}(\mathrm{Al}-\mathrm{Zn}-\mathrm{Mg}-\mathrm{Cu})$ alloys are mainly used for upper wing skin, and the balance of three main properties, (compression) yield strength, toughness and stress corrosion cracking (SCC) resistance [26], is crucial. Determination of SCC resistance is time consuming and hence routine assessment of SCC resistance of Al alloys is not economically viable. Instead, conductivity, which has been shown [27,28] to provide a measure for SCC resistance, has been used for routine assessment of SCC susceptibility. Thus, it is important to understand and predict the conductivity of heat-treated $7 \mathrm{xxx}$ alloys.

Several models or elements of models for the dependence of conductivity on composition and heat treatment have been presented by other workers [29,30]. These generally contain a description of the thermodynamics of the Al alloy system (e.g. based on regular solution model(s)), the kinetics of phase precipitation (e.g. based on JMAK kinetics [30]), and a model which gives conductivity as a function of the microstructure (e.g. [31]). In the present work we will present analyses of conductivity data of 7xxx alloys using AN and physically-based approaches, and compare the results achieved with the two methods. Both approaches were tested against a proprietary dataset which comprises conductivity data on a range of alloy compositions that broadly cover the high strength variants of the $7 \mathrm{xxx}$ series aluminium alloys. For each alloy, composition levels of $\mathrm{Zn}\left(\mathrm{x}_{\mathrm{Zn}, \mathrm{w}}\right), \mathrm{Mg}\left(\mathrm{x}_{\mathrm{Mg}, \mathrm{w}}\right), \mathrm{Cu}\left(\mathrm{x}_{\mathrm{Cu}, \mathrm{w}}\right), \mathrm{Zr}\left(\mathrm{x}_{\mathrm{Zr}, \mathrm{w}}\right)$, $\mathrm{Fe}\left(\mathrm{x}_{\mathrm{Fe}, \mathrm{w}}\right)$ and $\mathrm{Si}\left(\mathrm{x}_{\mathrm{Si}, \mathrm{w}}\right)$ in $\mathrm{wt} \%$ have been determined. The alloys were solution treated and 
subsequently aged for various times at a single temperature which is similar to the ones used for commercial T7 tempers. The electrical conductivity $\left(\sigma_{\mathrm{el}}\right)$ has been measured for 36 composition/ageing time variants.
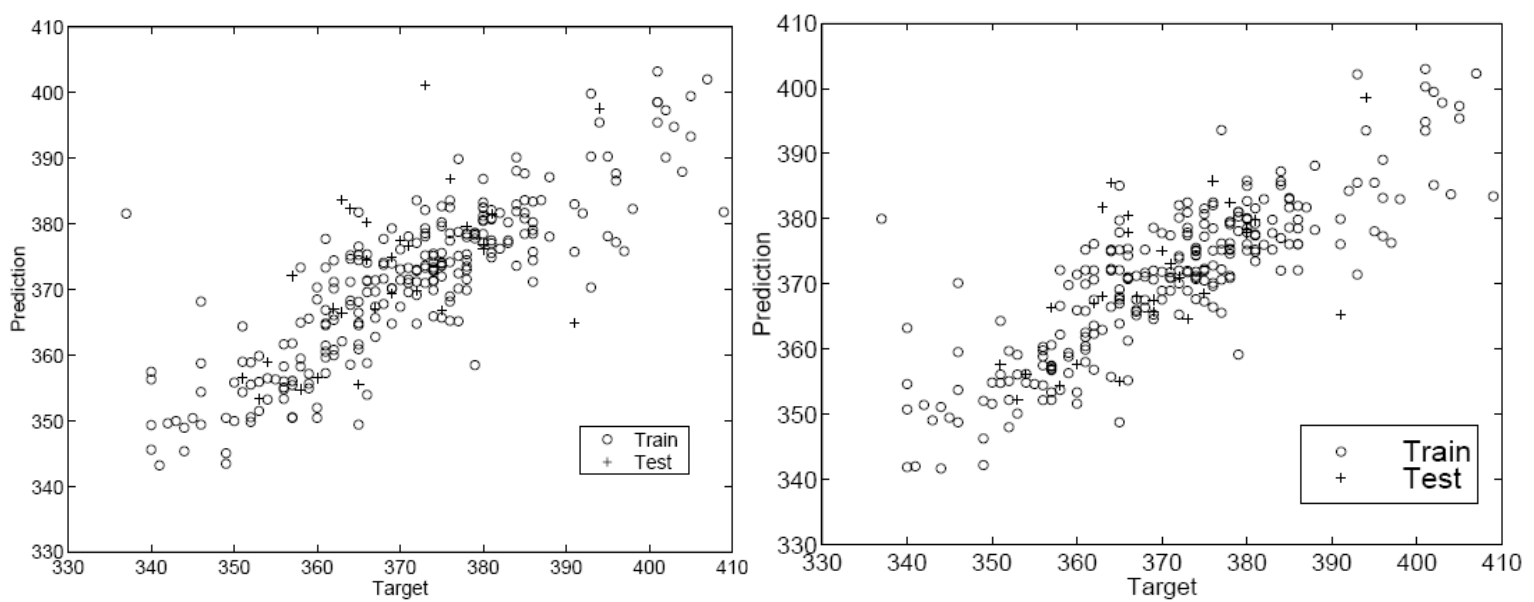

Fig. 3 Bayesian MLP and SVM target prediction plots for the dataset on the strength of 2024.

\section{AN modelling}

The dataset on the conductivity was analysed using NF and SVM modelling approaches. For adaptive numeric modelling approaches the present dataset is very small, and thus these approaches are tested in a very severe manner. SVM model construction proved to be particularly difficult because the trainingtest data split used in this algorithm effectively further reduces the number of datapoints available for model training. These results are discussed elsewhere [10], and in the present paper we will limit ourselves to the NF approach which, due to its reductionist nature, handles smaller datasets more consistently. The NF modelling approach identified a model which contains the only the $\mathrm{Mg}$ content and the ageing time. The dependence of conductivity on the $\mathrm{Mg}$ content is modelled as a linear function, whilst the time dependency is modelled as a bilinear (piecewise linear) function, see Fig. 4. The root-mean-squared-error (RMSE) achieved with this method is 0.61 IACS, i.e. conductivities are modelled with an accuracy of about $1.5 \%$.

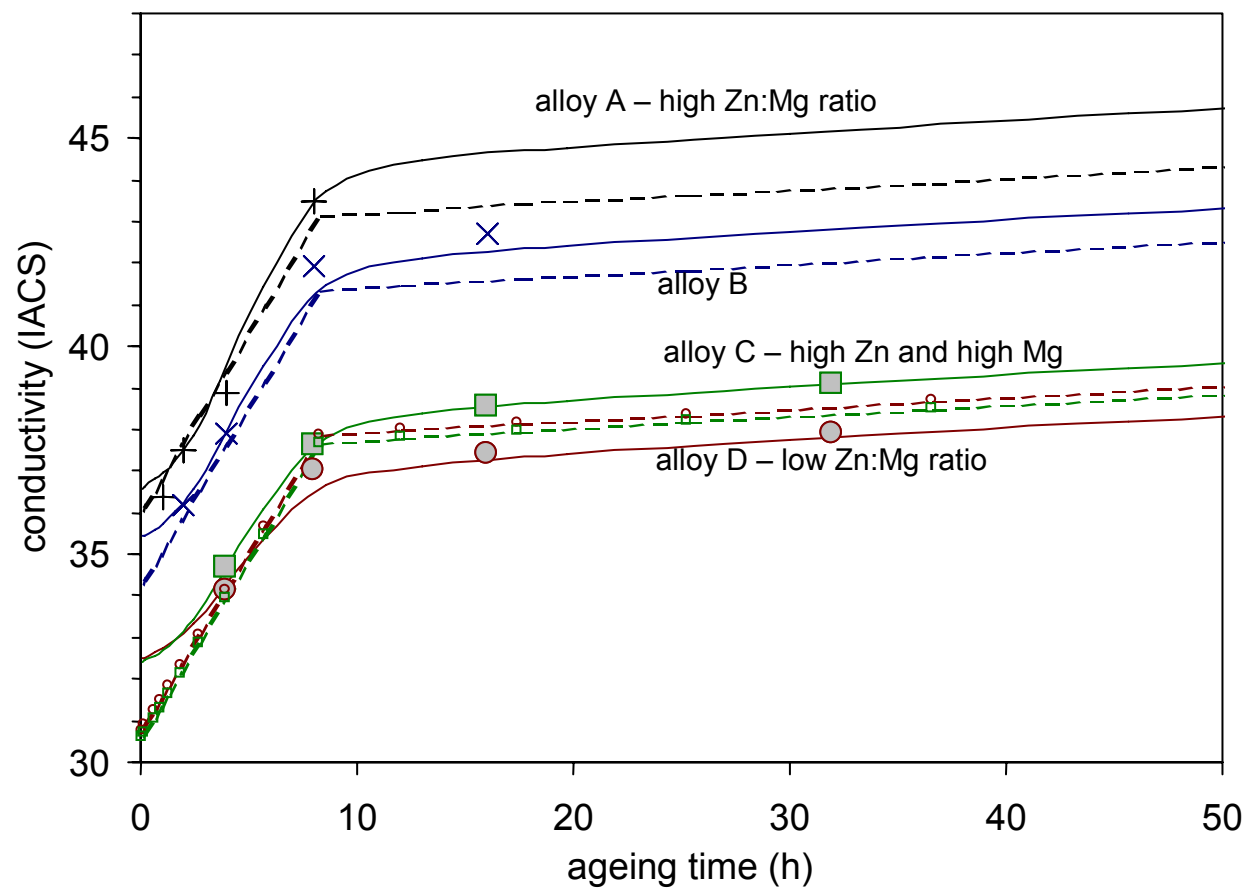

Fig. 4:

Conductivity of selected 7xxx alloys vs. ageing time at a typical T7 type ageing temperature. Alloy compositions are within the ranges of the main aerospace 7xxx alloys. Model results are from neurofuzzy model construction (--) and from a simplified physicallybased model (-) (see text). 
The dataset on the conductivity was further analysed using a hybrid approach, in which input composition data was pre-processed using some simple metallurgical and thermodynamic principles (i.e. regular solution models and fixed composition of the main precipitating phase, $\eta$ ). The use of these pre-processed variables allowed the construction of a marginally more accurate model with the NF approach (RMSE 0.59\%IACS). The model identified the excess $\mathrm{Mg}, \mathrm{x}_{\mathrm{Mg}, \mathrm{xs}}$, (the predicted amount of $\mathrm{Mg}$ left in solution after complete precipitation) and the ageing time as the main parameters influencing conductivity in these overaged alloys.

\section{Semi-empirical physical modelling}

To assess the applicability of a physically-based model to this dataset on conductivity of 7xxx alloys, a simplified model was derived using selected metallurgical knowledge and physical relationships. The model for conductivity considers the alloy to be divided into 2 zones with different conductivity. These zones are i) the grains (incorporating the matrix phase and coarse, undissolved particles) and ii) the solute depleted area (SDA) around the grain boundary. The shape of the grains is approximated as rectanguloids of a single size. The model further applies:

- simplified thermodynamic equations to model the amount of intermetallic phases (mainly $\mathrm{S}$ $\left(\mathrm{Al}_{2} \mathrm{CuMg}\right)[32,33]$ and $\mathrm{Al}_{7} \mathrm{Cu}_{2} \mathrm{Fe}: \mathrm{Mg}_{2} \mathrm{Si}$ and $\mathrm{T}$ phase (in $7 \mathrm{xxx}$ alloys a quaternary phase based on $\mathrm{Mg}_{3} \mathrm{Zn}_{3} \mathrm{Al}_{2} / \mathrm{CuMg}_{4} \mathrm{Al}_{6}$ ) are neglected because previous work [34,35,36] has indicated that they are either not present or only present in limited amounts in commercial 7xxx alloys),

- the Rayleigh-Maxwell equation for resistivity in a material with dispersed particles,

- the Starink-Zahra equation for precipitation [37,38],

- a one-dimensional diffusion equation to describe the growth of the SDA,

- Matthiesen's rule for the influence of dissolved elements on the resistivity $[39,40]$.

In the present model we will neglect the influence of the scattering of electrons by very small (nanometer sized) precipitates/zones [31]. It is thought that for the present overaged alloys this is a reasonable assumption. Further details of this model will be presented elsewhere [41].

The RMSE achieved by semi-empirical regression of this physically-based model to the available data (using 6 fittable parameters) is $0.43 \% \mathrm{IACS}$, and model predictions for selected alloys are presented in Fig. 4. It should be noted that $\rho_{\mathrm{Al}}$ obtained from the fitting procedure $\left((58 \% \mathrm{IACS})^{-1}\right)$ agrees reasonably well with the literature value for conductivity in pure Al (62\%IACS) when the effect of minor alloying elements $(\mathrm{Zr}, \mathrm{Ti})$ and impurities $(\mathrm{Fe}, \mathrm{Si})$ are taken into account. Conversely, the values of the proportionality constants in Matthiesen's rule, $r_{M g}, r_{Z n}$ and $r_{C u}$, as determined in the fitting procedure deviate considerably from the ones determined from single phase binary Al-Mg, $\mathrm{Al}-\mathrm{Zn}$ and $\mathrm{Al}-\mathrm{Cu}$ alloys $[28,42]$.

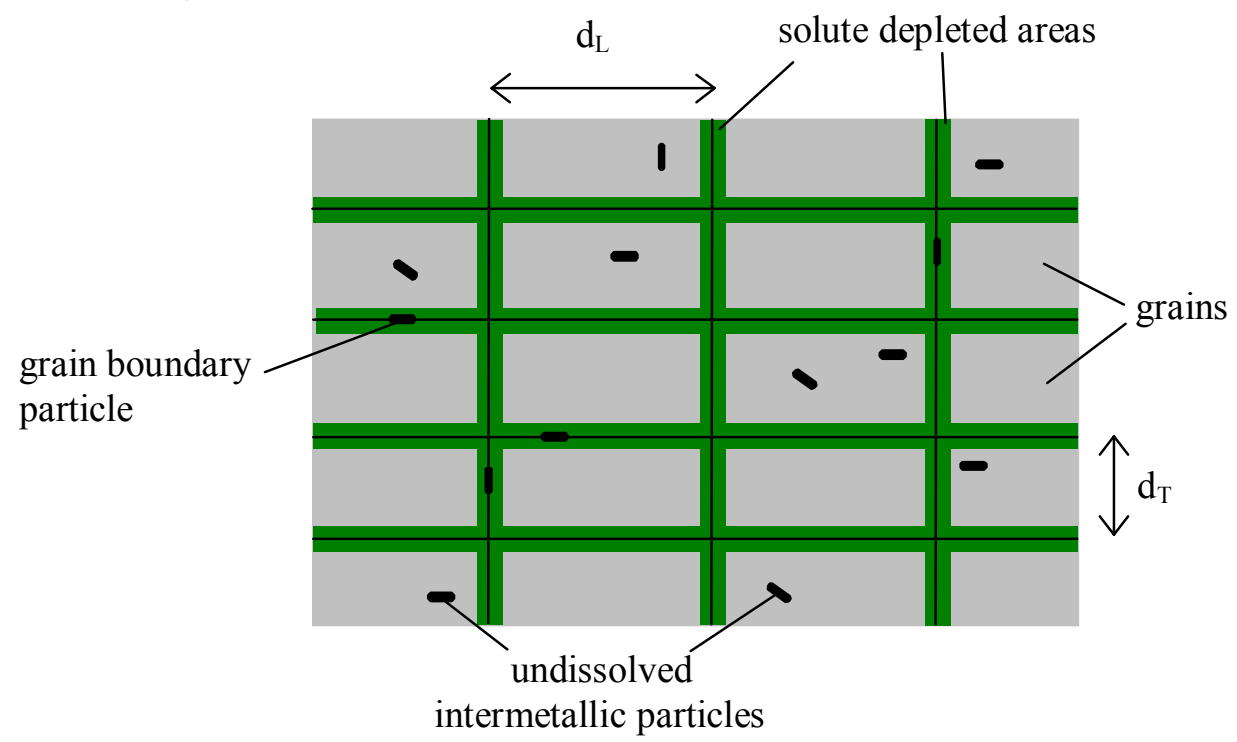

Fig. 5

Schematic drawing of the alloy structure considered in the model for conductivity. The grains are rectanguloids and the SDAs around the grain boundary provide connected conductivity paths in the shape of plates. 


\section{Comparison of modelling approaches}

In Table 1 statistical parameters for the presented model approaches are summarised, and compared with simple linear regression methods. The table illustrates that for the present conductivity data, the physically-based model is more accurate than the model constructed by the NF approach, although more fitting parameters were used by the semi-empirical physical model. Whilst the statistics in Table 1 are the main points to consider in deciding the relative success of the modelling methods, the present results show some additional interesting points related to modelling approaches. Firstly, in the NF model construction the influence of $\mathrm{Zn}$ and $\mathrm{Cu}$ on the conductivity was determined to be not statistically significant, which leads, for example, to the inaccurate prediction of nearly identical values for predicted conductivity for the alloys $\mathrm{C}$ and $\mathrm{D}$ in Fig. 4 (alloys $\mathrm{C}$ and $\mathrm{D}$ have nearly identical $\mathrm{Cu}$ and $\mathrm{Mg}$ contents). This contrasts with the analytical model, in which $\mathrm{Zn}$ content is included in a convoluted way, for instance through the formation of precipitates with a fixed $\mathrm{Zn}: \mathrm{Mg}$ ratio. It appears that the latter approach is more successful. Further, inclusion of simple metallurgical and thermodynamic ideas in data pre-processing prior to NF modelling to arrive at a hybrid approach has been successful in improving the accuracy of the models constructed (see also Ref. [8]). To further assess model performance, the extrapolation and interpolation accuracies of the two models were assessed by fitting the model to a training set from which 4 data points at extremes (i.e. high solute contents or low ageing times) or data points at average values of input parameters were excluded. Results have shown that interpolation and extrapolation ability of the two models varied according to the type of data omitted. Whilst the analytical model remained on average more accurate it showed no significantly better extrapolation or interpolation capacity as compared to the NF model. The general conclusion to be drawn from this comparison is that the NF approach has been able to construct a quite accurate model based on data alone, although some improvement in model performance can be obtained by taking account of physical understanding prior to NF modelling or by derivation of a semi-empirical physical model.

\begin{tabular}{|c|c|c|c|}
\hline Model Type & $\begin{array}{c}\text { Fittable } \\
\text { parameters }\end{array}$ & $\begin{array}{c}\text { RMSE - training } \\
(\% \text { IACS })\end{array}$ & $\begin{array}{c}\text { RMSE - test } \\
(\% \text { IACS })\end{array}$ \\
\hline Linear Regression & 7 & 1.9 & 2.1 \\
\hline Model constructed with NF approach & 5 & 0.54 & 0.61 \\
\hline Hybrid physical/NF model [8] & 5 & 0.48 & 0.59 \\
\hline Semi-empirical physical model & 6 & 0.39 & 0.42 \\
\hline
\end{tabular}

Table 1 Statistics of various modelling approaches to modelling of a database of conductivity data for $\mathrm{Al}-\mathrm{Zn}-\mathrm{Mg}-\mathrm{Cu}-\mathrm{Zr}$ alloys.

\subsection{Case 3: Strength of Al-Li based alloys and Metal Matrix Composites (MMCs).}

In aerospace applications $\mathrm{Al}-\mathrm{Cu}-\mathrm{Mg}$ based alloys are still the first choice for most damage tolerant applications. Al-Li based alloys (209x, 809x) have been developed as stiffer, lightweight alternatives to the incumbent Al-Cu-Mg-based alloys. Additionally, Al-Li MMCs have been considered for their excellent specific stiffness. Various issues however limit the widespread application of Al-Li based alloys and MMCs. For instance, it has been realised that in their damage tolerant (i.e. underaged condition) Al-Li based alloys undergo a further hardening process when exposed to moderately elevated temperatures (of the order of 50 to $80^{\circ} \mathrm{C}$ ) [43,44,45]. The concomitant loss in toughness [44] poses a serious problem for many of the potential applications for aluminium-lithium alloys. There have been various suggestions for the causes of this decrease in toughness [44,46,47], however the relative contributions of GPB zones and $\delta$ ' phase to the strengthening of these alloys, as well as the influence of precipitate free zones in Al-Li based MMCs has not been quantified.

In the present comparison of modelling approaches we will present results on the modelling of the strength of five $\mathrm{Al}-\mathrm{Li}-\mathrm{Cu}-\mathrm{Mg}$ based alloys and $\mathrm{SiC}$ reinforced alloys using data on the microstructure 
as obtained from DSC and TEM experiments. The database contains a total of 21 alloy-ageing treatment combinations, and 12 input parameters: the sizes of $\delta^{\prime}$ and $\mathrm{S}^{\prime}$ phases, volume fractions of $\delta^{\prime}$, $\mathrm{S}^{\prime}$ and GPB zones, size of the PFZ around $\mathrm{SiC}$ particles and $\mathrm{Li}, \mathrm{Cu}, \mathrm{Mg}, \mathrm{Zr}$ and $\mathrm{SiC}$ contents.

\section{AN modelling}

By applying the AN approaches to the present, very small dataset they are severely tested in a manner that they are generally not designed for. Notwithstanding this disadvantage, the NF and the SVM approaches were able to produce reasonable modelling accuracies (see Table 2). The NF approach identified the volume fraction of $\delta^{\prime}, f_{\delta}$, the diameter of $\mathrm{S}^{\prime}$ and the $\mathrm{Cu}$ content as the most influential parameters, whilst the SVM approach identified univariate terms for Li content and the diameter of $\mathrm{S}^{\prime}$, along with a bivariate term combining $f_{\delta^{\prime}}$ and the Li content, and one combining the $\mathrm{Zr}$ and $\mathrm{SiC}$ contents.

\section{Physically-based modelling}

The physically-based model used in the present work is derived taking into account all the relevant published work which goes back to 1940s with ground breaking work by Mott and Nabarro [1] and Orowan [2]. More recent substantial contributions in this field are for instance those by Ardell [48] and Nembach and Neite [49], and various other strengthening mechanisms, like grain strengthening, dislocation strengthening, solid solution strengthening and strengthening by inclusions, have been the subject of earlier work (see e.g. Refs. [50,51,52,53] and references therein). Hence, there exists a considerable body of work on modelling independent contributions of strength in alloys and composites but predictive modelling of properties of complex alloys and composites requires superposition of these contributions. This type of complex strength modelling has been performed, to a varying degree of detail, for several monolithic precipitation hardened alloys $[3,4,5,25,52,54,55]$, and recently a detailed model of strengthening in precipitation hardened metal matrix composites (MMCs) has been reported by the present authors $[56,57,58]$. In the strength modelling approach adopted in the present paper, the strengthening of MMCs and monolithic alloys are ascribed to five mechanisms:

i. Precipitation strengthening, which involves strengthening of grains due to GPB zones, $\delta$ ' $\left(\mathrm{Al}_{3} \mathrm{Li}\right)$ phase and $\mathrm{S}^{\prime}\left(\mathrm{Al}_{2} \mathrm{CuMg}\right)$ phase $[59,60]$, with a small contribution due to $\beta^{\prime}\left(\mathrm{Al}_{3} \mathrm{Zr}\right)$ dispersoids.

ii. Solution strengthening, which involves strengthening of the Al-rich phase by dissolved $\mathrm{Li}, \mathrm{Cu}$ and Mg atoms.

iii. Grain and subgrain strengthening, which involves strengthening due to hampering of dislocation movement by the presence of (sub-) grain boundaries.

iv. Dislocation density which involves the strengthening of the Al-rich phase grains due to impeding of dislocation movement by the presence of dislocations, which have formed as a result of prior plastic deformation; in MMCs the main cause for prior plastic deformation is the thermal misfit of ceramic inclusions, and

v. Load transfer between matrix and reinforcement in MMCs.

Some selected results of the modelling are presented in Fig. 6. (For further results see Refs. $[56,57,58]$.) The accuracy of the modelling is presented in Table 2. It is further noted that as part of the physically-based approach modelling of the microstructure evolution with ageing time has been performed, and this model can be applied to predict the continuous change in properties as well as extrapolations to longer ageing times as depicted in Fig. 6.

\section{Comparison of modelling approaches}

Table 2 shows that, on this small dataset, the physically-based approach outperforms the AN approaches. This indicates that the elements of the physically-based model presented are sound and that, in general, strength modelling of complex alloys can be successfully pursued using a physicallybased approach. It must however be emphasised that the amount of data for the NF and SVM model training was clearly well below that which may be expected to reasonably represent a problem of this complexity and the physically-based model required extensive experimental $[58,59,60]$ and analytical [56,57] development. 


\begin{tabular}{|c|c|}
\hline & RMSE - test $(\mathrm{MPa})$ \\
\hline NF Modelling & $24^{*}$ \\
\hline SVM Modelling & 41 \\
\hline Physically-based model & 16 \\
\hline
\end{tabular}

* Test error for the NF model obtained from a leave-one-out cross-validation test (see e.g. [8]). This test will somewhat underestimate the true testing error.

Table 2 Statistics of various modelling approaches to modelling of a database of strength data for $\mathrm{Al}-\mathrm{Zn}-\mathrm{Mg}-\mathrm{Cu}-\mathrm{Zr}$ alloys.

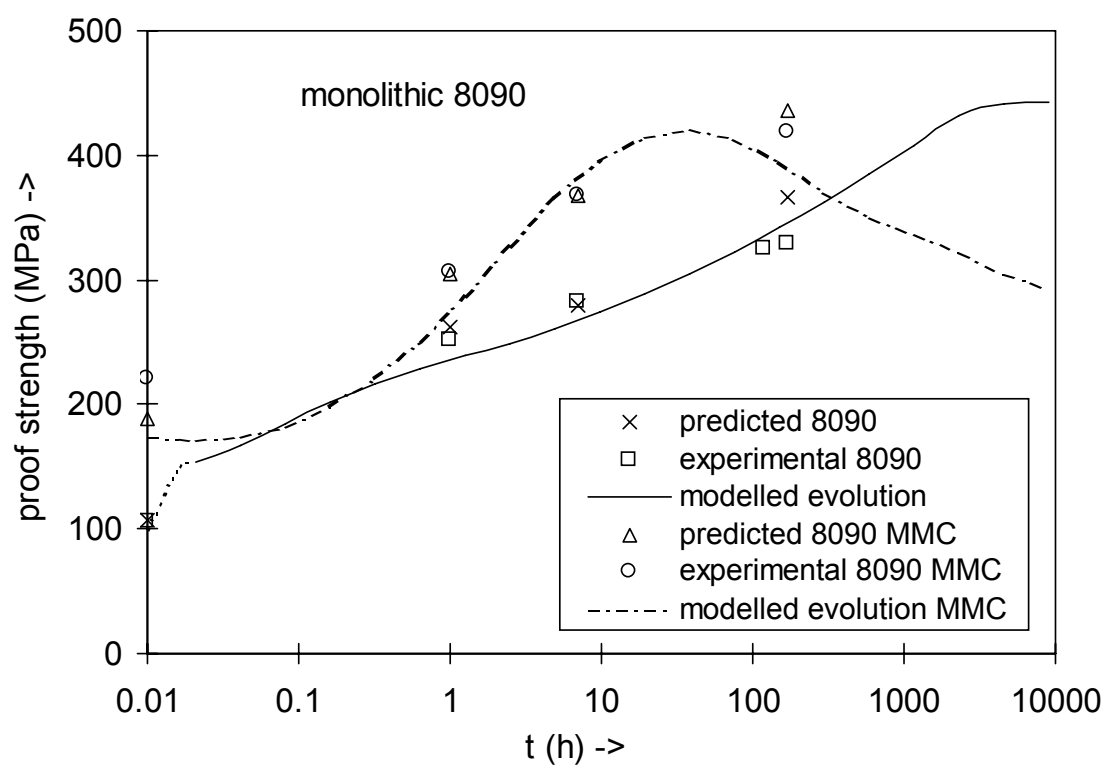

Fig. 6

Predicted and modelled strength (physically based model) of the 8090 monolithic alloy and the $8090 \mathrm{MMC}$ during ageing at $170^{\circ} \mathrm{C}$. The continuous evolution and extrapolation (lines) were modelled by combining the microstructure evolution model outlined in Ref. [59] and the physically based model.

\subsection{Case 4: Modelling toughness of high strength Al based alloys.}

The fracture toughness of high strength aluminium alloys is known to depend on many parameters, including flow strength, work hardening, slip character, dispersoid content, intermetallic content, grain structure, grain boundary structure and microstructural anisotropy [61]. Various models have been put forward in the literature to predict the influence of microstructural or mechanical parameters on the fracture toughness, however, these models generally consider only a selection of the parameters that are known to be important. For instance, assuming unstable crack extension proceeds when crack tip opening reaches the length of the unbroken ligaments separating cracked inclusions, the following expression may be derived $[62,63]$ :

$$
K_{I C} \approx\left\lfloor 2 \sigma_{y} E\left(\frac{\pi}{6}\right)^{1 / 3} D\right]^{1 / 2} f_{V}^{-1 / 6}
$$

where $D$ is the diameter of the large inclusions, $f_{V}$ is their volume fraction, $\sigma_{y}$ and $E$ are the yield stress and Young's modulus respectively, and $K_{I C}$ is the plane strain fracture toughness. This model has been shown to give a reasonable prediction of the effect of the volume fraction of inclusions for constant yield strength and constant particle size in a number of systems, but does not agree with experimental results concerning the influence of yield stress. In an earlier model, Hahn and Rosenfield [64] considered the effects of plastic deformation in a zone emanating from the crack tip, particularly in terms of the work-hardening exponent. Garrett and Knott [65] reviewed the derivation of this model leading to the relationship:

$$
K_{I C} \approx \sqrt{\frac{2 C E \varepsilon_{c}^{*} \sigma_{y} n^{2}}{1-v^{2}}}
$$


where $C$ is a constant, $\varepsilon_{c}{ }^{*}$ is the critical crack tip strain at which unstable propagation occurs, $n$ is the work hardening exponent and $v$ is the Poisson ratio; $\varepsilon_{c}{ }^{*}$ is taken to be a function of the volume fraction and distribution of void nucleating particles [66]. The predicted $n \sqrt{ } \sigma_{y}$ dependency of the fracture toughness for a constant distribution of particles has been shown to provide a reasonable description of toughness behaviour as a function of ageing between under- and overaged conditions (i.e. varying yield strength) for several Al-based alloys (data for 7075 is presented in Ref. [67]). Also the use of a critical stress criterion to describe the decohesion of the interface between the matrix and the dispersoids leads to a $n \sqrt{ } \sigma_{y}$ dependency of the fracture toughness [67]. Whilst these models consider only random distributions of particles Ehrstrom et al. and Achon et al. showed that in order to model anisotropic toughness, distributions of particles should be taken into account $[68,69]$.

In addition to transgranular failure processes (voiding at coarse intermetallic particles or void sheet formation at dispersoid particles), fracture in high strength aluminium alloys can also proceed by a ductile intergranular mechanism, which may depend on void initiation at boundary precipitates, strain localisation within soft precipitate free zones (PFZ) and stress/strain concentration at slip band/gb intersections [70], all of which are strongly dependent on ageing condition. A model for grain boundary failure assuming that all plastic deformation is entirely restricted to the PFZ region, has for example been derived by Hornbogen and Gräf [71].

Whilst the above models all have been successful in analysing selected aspects of toughness behaviour, a comprehensive model is, apparently, not yet available. It is considered that, as yet, microstructuretoughness relations are too complex to allow the development of a full physically-based model. For cases where large production databases are available modelling of toughness for specific alloys using AN modelling (specifically SVM-based) may be pursued [11], with models obtained to date achieving RMSE values of the order of $1.5 \mathrm{MPa} \sqrt{\mathrm{m}}$ in measured fracture toughness values for processing databases with up to 14 input variables (covering composition, thermo-mechanical processing data etc.).

\section{$4 \quad$ Future challenges for modelling.}

The examples presented in the previous section illustrate how different approaches to modelling can be successfully used to interpret available datasets of different sizes and to predict properties of alloys for different composition/processing combinations. The examples cover the full range from complete physically-based models which do not rely on fittable parameters (section 3.3), to full adaptive numeric modelling approaches on large datasets. Also hybrid approaches were presented, i.e. AN modelling approaches which make use of metallurgical insights to pre-process input data to obtain models with improved accuracy and/or transparency. These results should be considered within the context of the wider field of approaches used for physically-based modelling of the properties of metallic alloys, which further contains important techniques such as finite element (FE) modelling (for mechanical properties) and thermodynamic modelling [24,29] (for prediction of phases and phase diagrams). All these modelling approaches have their own specific benefits and limitations. On this point we have already noted that for large datasets with complex and hard-to-interpret properties, AN modelling will produce the best results. As another example we may notice that in order to predict casting, homogenising and solution treatment processes, thermodynamic modelling is an indispensable technique. The main challenges for the future lie both in the further development of individual techniques, as well as in the combination of the different modelling approaches to arrive at integrated approaches that are adapted to the properties being modelled, the data available and the economic/time constraints put upon R\&D. 


\section{Conclusions}

- Recent advances in physically-based and adaptive numeric modelling have made them indispensable techniques in analysing and predicting properties of metallic alloys. They play an important and increasing part in alloy development and process optimisation.

- Within the two main categories (physically-based and adaptive numeric) various techniques exists and hybrid approaches are also being developed.

- Selection of modelling approaches is driven by a range of factors, such as size of database available (large databases are best analysed using adaptive numeric modelling approaches), and availability of prior physical understanding (the latter benefiting physically-based approaches).

- Data on processing-conductivity, processing-strength and processing-toughness relations in 7xxx alloys can all be analysed successfully provided the most appropriate modelling approach is selected. The same holds for processing-property and microstructure-property relations in other alloys studied in this paper.

\section{Acknowledgements}

Technical discussions of elements of the present work with Drs. T. Warner, J.-C. Ehrström and C. Sigli (Pechiney CRV), J. Newman and A. Morris (British Aluminium Plate), H. Hollroyd (Luxfer Gas Cylinders), P. Pitcher (DERA, Farnborough) and B. Noble (University of Nottingham) are gratefully acknowledged. Provision of alloys and confidential data by British Aluminium Plate, DERA, Pechiney CRV and British Aerospace is gratefully acknowledged. The authors are further indebted to Dr. Steve Gunn and other members of the Image Speech and Intelligent Systems (ISIS) Group of the University of Southampton, for their invaluable work on the development of ANM approaches, some of which are reviewed and applied in this paper. Assistance of O.P. Femminella, S. Christensen, J.S. Kandola and Xiaomei Li with AN and physically-based modelling is gratefully acknowledged.

\section{References}

1 N.F. Mott and F.R.N. Nabarro, Proc. Phys. Soc. 52 (1940) 86.

2 E. Orowan, in Symposium on Internal Stresses in Metals and Alloys, Session III Discussion (Institute of Metals, London, U.K., 1948) p. 451.

3 J.C. Huang and A.J. Ardell, Mater. Sci. Eng. A104 (1988) 149.

4 J.C. Huang and A.J. Ardell, Acta Metall.36 (1988) 2995.

5 H.R. Shercliff and M.F. Ashby, Acta Metall. Mater.38 (1990) 1789.

6 T.J. Warner, R.A. Shahani, P. Lassince and G.M. Raynaud, in Proc of the $3^{\text {rd }}$ ASM conference on "Synthesis, Processing and Modelling of Advanced Materials", Paris, June 1997

7 J.S. Kandola, S.R. Gunn, I. Sinclair and P.A.S. Reed, submitted to Machine Learning.

8 O.P. Femminella, M.J. Starink, M. Brown, I. Sinclair, C.J. Harris and P.A.S. Reed, ISIJInt. 39 (1999) 1027.

9 H.K.D.H. Bhadeshia, ISIJ Int. 39 (1999) 933

10 O.P. Femminella, M.J. Starink, C.J. Harris, S.R. Gunn and P.A.S. Reed, Proc. ICAA7, 9-14 April 2000, Charlottesville, Virginia, USA (these proceedings)

11 S. Christensen, J.S. Kandola, O.P. Femminella, S.R. Gunn, P.A.S. Reed and I. Sinclair, Proc. ICAA7, 9-14 April 2000, Charlottesville, Virginia, USA (these proceedings)

12 R.M.V. Pidaparti and M.J. Palakal, AIAA Journal 36 (1998) 1300.

13 A. Forcellese, F. Gabrielli, and R. Ruffini, J. Mater. Process. Techn. 80 (1998) 493.

14 S.M. Roberts, J. Kusiak, Y.L Liu, A. Forcellese and P.J. Withers, J. Mater. Process. Techn. 80 (1998) 507.

15 M.S. Chun, J. Biglou, J.G. Lenard and J.G. Kim, J. Mater. Process. Techn. 86 (1999) 245.

16 Y.S. Tarng, H.L. Tsai, S.S. Yeh, Int. J Mach. Tools Manuf. 39 (1999) 1427.

17 J. Hadamard, Lectures on the Cauchy Problem in Linear Partial Differential Equations (Yale University Press, 1923)

18 V. Vapnik, The Nature of Statistical Learning Theory (Springer-Verlag Publishers, 1995)

19 M. Brown and C. Harris, Neurofuzzy Adaptive Modelling and Control (Prentice-Hall, 1994)

20 K.M. Bossley, PhD thesis, University of Southampton (1997).

21 J.M. Schooling, M. Brown and P.A.S. Reed, Mater. Sci Eng. A260 (1999) 222.

22 J.M Schooling and P.A.S. Reed, Proc. $8^{\text {th }}$ Int. TMS Conf. on Superalloys, Seven Springs, USA (1996) 409.

23 D.J.C. MacKay, Bayesian Non-linear Modelling for the Prediction Competition. ASHRAE Transactions: Symposia, OR-94-17-1 (1994) 1053. 
24 N. Saunders, Proc of ICAA-5, Mater. Sci. Forum 217-222 (1996) 667.

25 A.W. Zhu, A. Csontos and E.A. Starke, Acta Mater. 47 (1999) 1713.

26 N.J.H. Hollroyd, Proc. of Environment-Induced Cracking of Materials, 1989, 311.

27 T.C. Tsai and T.H. Chuang, Corrosion 52 (1996) 414.

28 W. Hepples, PhD Thesis, University of Newcastle upon Tyne, 1987

29 C. Sigli, L. Maenner, C. Sztur and R. Shahani, Proc. ICAA-6, Toyohashi, Japan, July 5-10, 1998, p. 87

30 D.H. Bratland, Ø. Grong, H. Shercliff, O.R. Myhr and S. Tjøtta, Acta Mater. 45 (1996) 1.

31 P. Guyot and L. Cottignies, Acta Mater. 44 (1996) 4161

32 A.J. Morris, R.F. Robey, P.D. Couch and E. De los Rios, Proc. ICAA-5, Mater. Sci. Forum 242 (1997) 181

33 P. Sainfort, C. Sigli, G.M. Raynaud and Ph. Gomiero, Proc. ICAA-5, Mater. Sci. Forum 242 (1997) 25

34 Xiaomei Li and M.J. Starink, Proc of the $5^{\text {th }}$ PostGraduate Conf. in Engineering Materials, 1 Oct 1999, Winchester, U.K., (University of Southampton, U.K., 1999)

35 Xiaomei Li and M.J. Starink, Mater. Sci. Forum, 2000, vol. 331-337, pp. 1071-1076

36 J.A. Wert, Scr. Metall. 15, (1981) p. 445

37 M.J. Starink and A.-M. Zahra, Thermochim. Acta 292 (1997) 159

38 M.J. Starink and A.-M. Zahra, Phil. Mag. A 77 (1998) 187

39 J.O. Linde, Ann. Physik 10 (1931) 52

40 R.H. Brown and L.A. Willey, in Aluminium: Physical Properties, K.R. van Horn, ed., ASM, Ohio, 1967

41 M.J. Starink and Xiaomei Li, Metall Mater Trans A, 2003, vol 34A, 899-911

42 J.E. Hatch (ed.), Aluminium - Properties and Physical Metallurgy (ASM International, Metal Park, Ohio, 1984)

43. C.J. Peel, in Proc. Al-Li alloys VI, eds M. Peters and P.J. Winkler, Oberursel, Germany, 1992, DGM Metallurgy Information, 1259-1270.

44. W.J. Vine, G.R. Sutton and H.J. Price, in Proc. of ICAA-6, Toyohashi, Japan, July 5-10, 1998, p. 1973

45 H. Octor, E. Bouchaud and G. Lapasset, Recherche Aerospatiale 5-6 (1996) 393

46. S.P. Lynch, A.R. Wilson and R.T. Byrnes, Mater. Sci. Eng. A172 (1993) 79.

47. B. Noble, S.J. Harris, and K. Dinsdale, Proc. of ICAA-4, Atlanta, GA, September 11-16, 1994, p. 460.

48 A.J. Ardell, Metall. Trans. A.16 (1985) 2131.

49 E. Nembach and G. Neite, Prog. Mater. Sci. 29 (1985) 177.

50 W.S. Miller and F.J. Humphries, in Fundamental Relationships Between Microstructure \& Mechanical Properties of MMCs, ed. P.K. Law and M.N. Gungor, TMA, Warrendale, Pa., USA, 1990, p. 157.

51 D.C. Dunand and A. Mortensen, Acta Metall. Mater. 39 (1991) 127.

52 P. Gomiero, Y. Brechet, F. Louchet, A. Tourabi and B. Wack, Acta Metall. Mater. 40 (1992) 857.

53 U.F. Kocks, Metall. Trans. A.16 (1985) 2109.

54 C. Schlieser and E. Nembach, Acta Metall. Mater. 43 (1995) 3983.

55 A.W. Zhu and E.A. Starke, Acta Mater 47 (1999) 3263.

56 M.J. Starink, P. Wang, I. Sinclair and P.J. Gregson, Acta Mater. 47 (1999) 3855.

57 M.J. Starink and S. Syngellakis, Mater. Sci. Eng. A 270 (1999) 270.

58 M.J. Starink, A.J. Hobson and P.J. Gregson, Mater. Sci. Forum, 2000, vol. 331-337, pp. 1321-1326.

59 M.J. Starink, P. Wang, I. Sinclair and P.J. Gregson, Acta Mater. 47 (1999) 3841.

60 M.J. Starink and P.J. Gregson, Scr. Metall. Mater. 33 (1995) 893.

61 D. S. Thompson, Metall. Trans. 6A (1975) 671.

62 G. T. Hahn and A. R. Rosenfield, Metall. Trans. 6A (1975) 653.

63 J. R. Rice and M. A. Johnson, Inelastic Behavior of Solids (New York: McGraw-Hill, 1970) p. 641.

64 G. T. Hahn and A. R. Rosenfield, ASTM, STP 432 (1968) 5.

65 G. G. Garrett and J. F. Knott, Metall. Trans. 9A (1978) 1187.

66 D. Broek, Eng. Fract. Mech. 5 (1973) 55.

67 C.Q. Chen and J. F. Knott, Met. Sci. 15 (1981) 357.

68 J.C. Ehrstrom, P. Achon, J.F. Hebert and A. Pineau, Proc of ICAA-5, Mater. Sci. Forum 217 (1996) 1539.

69 P. Achon, J.C. Ehrstrom and A. Pineau, J. Phys. IV 6-C6 (1996) 3

70 A. K. Vasudevan and R. D. Doherty, Acta Metall. 35 (1987) 1193.

71 M. Gräf and E. Hornbogen, Acta Metall. 25 (1977) 877. 